\title{
Towards Online Delivery of Process-Oriented Guided Inquiry Learning Techniques in Information Technology Courses
}

\author{
Jarrod Trevathan \\ Griffith University \\ j.trevathan@griffith.edu.au \\ Trina Myers \\ James Cook University \\ trina.myers@jcu.edu.au
}

\begin{abstract}
Process-Oriented Guided Inquiry Learning (POGIL) is a technique used to teach in large lectures and tutorials. It invokes interaction, team building, learning and interest through highly structured group work. Currently, POGIL has only been implemented in traditional classroom settings where all participants are physically present. However, advances in online learning technologies have prompted increases in the popularity of flexible delivery and distance education courses. Therefore, teaching methodologies need to reflect the changing student demographic. This paper describes a preliminary approach for adapting POGIL techniques for use in tertiary courses delivered online. We discuss the current technologies that can be used for teaching online and contrast their suitability for POGIL. A teaching format is presented using Web 2.0 technologies (a wiki, blogs, and social networking) as a starting point for POGIL. We describe how an online third year Information Technology subject adopted these technologies for a condensed implementation of POGIL.
\end{abstract}

\section{Keywords}

POGIL, online delivery, student interaction, Web 2.0, Facebook.

\section{Introduction}

Online education tools are rapidly changing the way students learn and their expectations towards how teachers should deliver subject-related content. Many tertiary institutions use the term "flexible delivery" to describe courses using off-campus modes of teaching. Online technologies are at the core of flexible delivery courses and facilitate material provision, student collaboration, and subject administration. While there is much hype surrounding the new technologies, the core challenge for education practitioners is how to employ the technologies in a manner that is engaging and results in measurably successful learning outcomes for students.

There is an abundance of literature that discusses education theory and methodologies for improving student outcomes (e.g., Constructivism (Bauersfeld, 1995; Ben-Ari, 1998), Instructional Design (Sweller et al, 1998), Engagement Theory (Kearsley \& Shneiderman, 1998), etc.). However, most of these relate to a traditional classroom setting where the students and the teacher are all physically present. Therefore, it is not always possible to transition or apply these methodologies in the context of a purely online teaching environment (i.e., no face-to-face interaction amongst the lecturer and students or amongst the students themselves). 
This paper concentrates on the Process-Oriented Guided Inquiry Learning (POGIL) teaching methodology (D. Hanson \& Wolfskill, 2000; D. M. Hanson, 2006; POGIL, 2012). POGIL is a technique used to teach in large lectures and tutorials, which invokes interaction, team building, learning and interest through highly structured group work. Existing POGIL techniques have only been trialled in traditional classroom settings for teaching Chemistry (Farrell, Moog, \& Spencer, 1999; Moog \& Spencer, 2008). However, Myers, et al. (2012) recently adapted POGIL for use in Information Technology (IT) classes with encouraging results. The Myers, et al. (2012) study involved two subjects (networking and databases) taught over a two-year period. The study found that students' problem solving abilities, interpersonal skills and learning outcomes improved significantly. Over $85 \%$ of the students surveyed stated that they found POGIL beneficial to their studies.

This paper investigates how to implement POGIL in an online teaching environment for IT courses. We discuss the current online learning technologies and contrast their suitability for POGIL. A preliminary teaching framework is proposed that uses a wiki, blogs and social networking. Quantitative and qualitative evidence is presented from an online third year IT university subject that describes experiences from the teaching staff and students about the technologies and the preliminary group work format. POGIL was not implemented in a $100 \%$ pure form (i.e., using "highly structured" group work) as in studies by Myers, et al. (2012) and Douglas and Chui (2012). Instead, a condensed version of POGIL was employed to keep the study constrained to the learning outcomes and experiences in line with the technologies tested. The experiences presented in this paper lay the groundwork for how to aspire towards a full adaptation of POGIL online.

This paper is structured as follows: the following section provides a brief overview of the POGIL teaching methodology, the challenges faced for implementing POGIL in an online learning environment, online learning technologies and this study's goals. Next, we outline the technology platform and condensed POGIL teaching format used in a year IT subject. An anecdotal study follows of how successful the technology platform and teaching format were for engaging students and fostering an environment conducive to POGIL. The paper concludes with a brief summary and avenues for future work.

\section{Motivation for adopting POGIL for use in online courses}

\section{How POGIL Works}

POGIL is a method that uses highly structured group sessions where students work on processoriented tasks to derive answers as a collective. The students work together in groups of three or four and are assigned one of the following roles: Manager, Recorder, Presenter or Reflector (optional). The use of roles enforces accountability within the groups so the outcomes are peerdriven as opposed to instructor-driven where the motivation of completing tasks is to satisfy the instructor. Each role is dependent on the other roles, so students are accountable to their peers for the function they play (D. Hanson \& Wolfskill, 2000; D. M. Hanson, 2006; POGIL, 2012).

The Manager ensures that team members are fulfilling their roles, accomplishing the assigned tasks on time, and all group members are participating in the activities and understanding the concepts. The Recorder scribes the group's discussions and important aspects of the group's observations, insights and the significant concepts learnt. The Presenter delivers concise oral reports to the class. The Reflector observes group dynamics, behaviour and performance and may report to the group (or the class) about how well the group operates. 
The POGIL activities focus on core concepts and encourage a deep understanding of the course material through an exploration to construct understanding while developing higher-order thinking skills. The tasks include directed, convergent or divergent questions. Directed questions can be answered directly from the information provided, convergent questions require groups to reach a consensus of the solution and divergent questions can have a range of possible correct responses. To initiate POGIL in a subject, the class must first undergo a "building the POGIL culture" stage. This is a process whereby the students come to understand the benefits of using POGIL. This is achieved though fun exercises that prove effectively to students the advantages of shared information and collaborative learning. The students also learn the rules and roles of the "highly structured" group sessions, and the expectations for each session.

Once the POGIL culture has been established, students come to class prepared for POGIL group work. While there are numerous formats for how POGIL lessons can be structured, a common approach is to have a ten-minute lecture, then a breakaway POGIL session for five minutes. During the POGIL session, the students will work on an exercise related directly to the lecture content. The instructor mingles amongst the groups to gauge how the students are performing. At the end of the session, two or more groups are called on to report back to the class. The instructor then typically recaps and/or adds to the students' answers.

\section{The challenges for bringing POGIL into a purely online environment}

Adapting POGIL for use in an online environment presents many unique complications that differ from the study by (Myers et al., 2012). The main challenges this paper seeks to explore include:

Challenge 1: How to implement POGIL in a purely online environment in a manner that keeps students engaged - With online delivery students are not all in the same place at the same time. Therefore, the "real-time" nature of POGIL in a lecture theatre is lost. The interaction within the group will be staggered at best (i.e., tasks could take hours, days or even a week to complete compared to ten minutes in a normal lecture setting). This problem is exacerbated when dealing with students from multiple time zones around the globe.

Challenge 2: How to limit the time a convenor spends on material preparation and subject administration (particularly with a large class) - A trade-off must be reached between how much effort is required to initiate POGIL online versus the expected benefits to student learning.

Challenge 3: How to deal with differing Information and Communication Technology (ICT) skill sets and computing infrastructure amongst students - Some assumptions must be made about a student's basic computer skills and access to adequate ICTs. Ideally, an online POGIL session should be available for students across all academic disciplines. However, scholarly backgrounds and budgets for computing infrastructure will vary. Furthermore, international students less familiar with the English language may have difficulty with following instructions - particularly without face-to-face personal tutorage.

Challenge 4: How much reliance should be placed on technology (especially emerging technology) - Equipment invariably fails. A computer lab at a tertiary institution generally will not have all computers functioning. Without the technical support that is embedded within institutions, home computing paradigms are even more volatile. If students are responsible for their own equipment, the likelihood of flat batteries, corrupt hard drives, network outages and computer viruses are much higher and often occur at the most critical times. 


\section{The implications of online learning technologies on POGIL}

Web 2.0 technologies marked the transition of the World Wide Web from static content consumed by the end user to having the end user dynamically contributing and creating the content. Examples of Web 2.0 technologies include blogs, wikis, RSS feeds and podcasts. Such technologies have been dubbed "social software" as they facilitate collaboration and connectedness with relatively little technical expertise required on the user's behalf.

Most tertiary institutions now offer Learning Management Systems (LMS) such as Moodle and Blackboard. A LMS provides teaching staff and students with an ever-expanding range of tools for material dissemination, collaboration and assessment. An LMS is a good starting point for trialling POGIL online. However, a LMS cannot be seen as a "silver bullet" that can provide all of the functionality necessary for implementing POGIL. For example, many of the Web 2.0 tools contained within LMS are restrictive, and might only provide a subset of features that free vendors offer. Furthermore, students may be more familiar/comfortable using non-LMS tools. Therefore, a combination of commercial Web 2.0 and LMS technologies needs to be investigated.

Wikis appear to be an ideal tool for facilitating the group collaboration required for POGIL. A recent study by Davidson (2012) found wikis to be an effective platform for students to communicate and collaborate on a group project because they provide ubiquitous access to the project's organisation and version control. A wiki can be set up on Blackboard relatively easily. Each group member has "write" access to the group's wiki. Members can then add content when ready to the wiki and contribute to the group's answer. The good point is that not much computing infrastructure is needed (i.e., a low bandwidth Internet connection is sufficient).

Blogs are another simple Web 2.0 tool that allows individual students to post content online. There is also the ability for others to make comments on the blog owner's posts. Blogs are more individual than wikis in that posted content is read-only. Blogs also provide a chronological ordering of posts so that the history of edits can be viewed.

Social media is an extremely popular facet of Web 2.0. While LMS contain discussion boards for interactive conversations between students and teaching staff, commercial social networking sites (such as Facebook) are already very popular with students. Preliminary studies on using Facebook for teaching by Gray (2013) suggest that many students are more apt to check Facebook than go to the effort of periodically engaging with a discussion board on a LMS. Furthermore, Facebook's user interface is already familiar to many students, which may make Facebook a more suitable and engaging choice for hosting subject-based discussions.

There is the possibility of using video conferencing tools such as Skype and other collaborative tools such as Collaborate. However, for the purposes of this study, the initial platform for student interaction towards implementing POGIL was kept simple (Challenges 3 and 4).

The goals for this paper in terms of POGIL include:

1. Establish an engaging and interactive online community ("technology platform") conducive to POGIL group work (Challenges 1, 2, 3 and 4).

2. Use the technology platform to experiment with a condensed POGIL teaching format that can be expanded upon in future studies (Challenges 1 and 2). 


\section{Methodology - The trial online information technology course}

The trial course is an online information technology subject at Griffith University that covers information and content management. In Semester Two, 2012 (August - November), the curriculum covered three areas: Web 2.0, Information Architecture, and Content Management Systems. Subject materials were provided online via the Blackboard LMS (i.e., instructions, lecture slides, links to YouTube videos, and announcements).

\section{The condensed online POGIL format}

Figure 1 shows the technological platform that was used in the study. The platform consists of:

- A wiki as a central navigational point and progress tracker (hosted on a LMS);

- A series of student blogs (hosted on free blogging sites such as Wordpress and Blogger) divided into POGIL groups; and

- A Facebook group as a class discussion forum.

The remainder of this section describes how this platform was used to implement the online (condensed) POGIL teaching format.

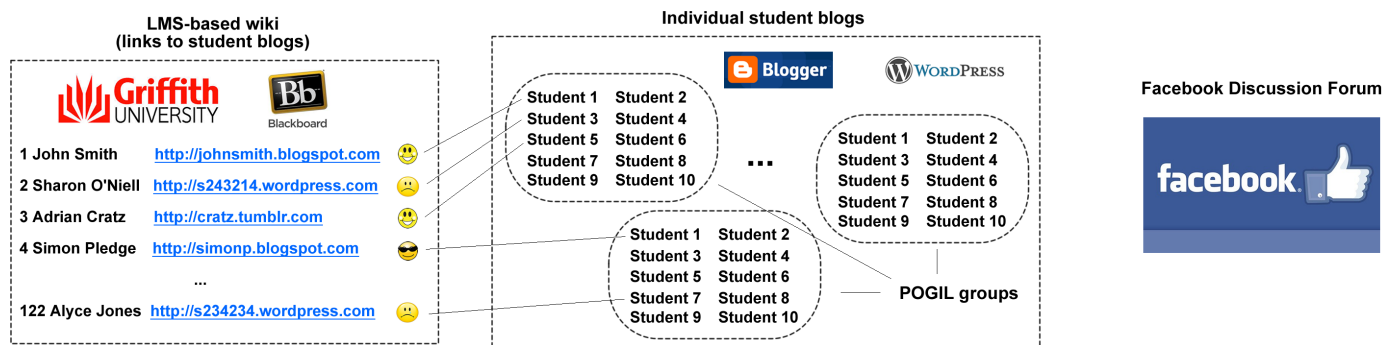

Figure 1. The technology platform using a wiki, individual student blogs, and Facebook

To undertake course content-related exercises, each student was tasked with establishing his/her own blog using a free blogging service. As individuals, students would then answer a series of questions from across four tasks using their blogs (referred to as Blog 1, Blog 2, Blog 3 and Blog 4 respectively). The blogs were publicly accessible so all students could see each other's work. The tasks were predominantly process-oriented convergent and/or divergent questions.

Without detracting from the main structure, POGIL was condensed to suit the online environment and subject learning outcomes. The main reason for the POGIL roles is to invoke peer accountability and group dependency. Students were divided into groups of ten instead of the standard POGIL membership of three or four with roles, due to the online environment and size of the class. Peer accountability and group dependency were maintained in this study with the expectation each student must add comments to their other group member's blogs. Marks were given for the overall blogs (i.e., an accumulation of the task responses and the group's comments), which effectively added the important layer of peer accountability without the need to delegate specific roles within the groups. The requirement to comment on other blogs promoted peer assessment and comments posted prompted self-reflection, which resulted in updates to the original blogs.

The blogs evolved with each iteration of the response-comment-update process. This evolution of the collaborative contributions to the blogs emulated the interaction found in face-to-face, realtime group work. Students responded much the same way as in face-to-face environment as they endeavoured to show they were significant participants in the group's outcomes. 
To reduce the administration burden on the teaching staff to keep track of everyone's external blog address, a central class wiki was set up via a LMS (Challenge 2). Students could use the wiki to add a hyperlink to their own blogs. Emoticons were used to provide students with relative feedback regarding their progress (e.g., a smiley face for being up-to-date or a teary face for being behind schedule). This served as a psychological prompt and a way to reduce administration by not having to provide specific feedback for each student. It was intended that student comments on blogs would provide more in-depth feedback, thereby reducing the load on the teaching staff.

A Facebook group was created for the subject to aid in establishing a community environment. A Facebook group allows existing Facebook users to sign up by making a request to the group's administrators (i.e., the teaching staff in this case). The group is "closed" so comments made within the group are not visible to those who are not part of the group. Furthermore, only basic information about group members is available, rather than what would occur if the members "friended" each other (Gray, 2013). The Facebook group was used to provide students with another channel to interact with each other, a way to familiarise themselves with others via profile pictures, and a mechanism to facilitate question asking and sharing to reduce the administration load on the teaching staff (Challenges 1,2, 3 and 4).

In terms of POGIL, the Facebook group did not involve any formal type of group work. The purpose of having a Facebook group for the course was to gather experience on how students reacted to social media in a teaching setting, establish what functionality in Facebook would be beneficial to administering POGIL, and to see how successful social media would be in fostering an online community and promoting engagement.

Table 1 illustrates the differences between the face-to-face POGIL employed by Myers, et al. (2012) and the condensed version of POGIL as it was implemented in this study. Firstly, the group sizes were ten in the online environment with no roles rather than the normal case of three to four with structured roles. Instead of roles, alternative methods were used to maintain the group dependencies and peer accountability. Similar to the Myers, et al. (2012) study, commenting on other students' blogs facilitated peer evaluation as would occur in POGIL. As the roles were not used, there was no need to engage in the "building the POGIL culture" exercises usually performed to introduce students to the group structure. Activities were not constrained by time with the online environment as they are in the classroom where tasks are limited to 5-10 minute deadlines. Finally, this study focused on being online, whereas the Myers, et al. (2012) study did not.

Table 1: Contrasting the online POGIL teaching format with the face-to-face POGIL format used in the Myers, et al. (2012) implementation

\begin{tabular}{lcc}
\hline & Face-to-face POGIL & Online POGIL Format \\
\hline Highly structured group work & Yes & No \\
Group size & $3-4$ & 10 \\
Peer accountability & Yes & Yes \\
POGIL style tasks & Yes & Yes \\
Building the POGIL culture & Yes & No \\
Time-constrained activities & Yes & No \\
Online & No & Yes \\
\hline
\end{tabular}




\section{Preliminary evidence of the effectiveness of using Web 2.0 technologies to build an online POGIL environment}

This section evaluates how conducive the online technology platform was to facilitating the condensed POGIL group work teaching format. Quantitative and qualitative evidence is presented from the perspectives of the teaching staff and the students respectively. Note that there is some overlap between the perspectives (that is, the staff and students felt or observed the same thing).

\section{Teaching Staff Observations}

There were two teaching staff directly involved with the course, and two other teacher staff that observed the course from a stakeholder perspective. The anecdotal observations below were gathered from informal discussions amongst the teaching staff.

\section{Blogs - Positive}

- The blogs served as an independent learning journal to track the progress of each student.

- Timestamps on postings can track the exact time and progress made on the work.

- The extent that students customised their blogs seemed to provide an indicator of their enthusiasm towards the course content.

\section{Blogs - Negative}

- Continual checking of student blogs was required to see what progress has been made. Given the class size (122 students) a spreadsheet tracking system was required and the help of a casual teaching staff member for six hours each week.

- Students can make inappropriate blog postings that are offensive or use bad language. A netiquette policy is required with enforcement through the assessment marking criteria.

- Students can disable the ability for others to comment on their blogs, which effectively excludes these students from interaction with other class members.

- The deadlines for blog postings were too flexible. There was a tendency for students to wait towards the end of the subject before making their postings, which affected the level of interaction these students had with the teaching staff and other students. Deadlines need to be more stringently enforced.

- Some students misunderstood the requirements for blogging and created a blogging engine from scratch rather than using an existing tool (under the belief that they would get bonus marks). This problem is perhaps more specific to IT or engineering students than other disciplines and can be addressed through refining the assessment specification.

\section{Class Wiki - Positive}

- The wiki reduced much of the administrative burden from the teaching staff.

- The use of emoticons to provide feedback on progress was highly successful. If a student received a teary face the majority of the time his/her blogging would come up-to-date within days. Students were also directed to high quality blogs that were indicated by a smiley face or a cool face with sunglasses.

\section{Class Wiki - Negative}

- There were students that did not follow the instructions and just wrote the URL rather than making a hyperlink to their blogs (which meant the teaching staff had to edit the wiki on the student's behalf).

- As all students have write permission for the wiki, there is the possibility to either inadvertently or maliciously remove or overwrite other students' blog addresses. Fortunately, this did not happen during this course. 


\section{Facebook - Positive}

- A lecturer can see which students have been viewing which comments/postings and can therefore decide which areas require priority.

- Bad or misleading postings can be addressed immediately, thereby stemming any damage.

- A group administrator in Facebook can moderate comments and remove anything offensive.

- When a student has correctly answered a query posted by another student, the teaching staff can "Like" the comment. This removes the need for the teaching staff to repeat the information and students can see that the response has been endorsed.

- There is a student perception that the teaching staff are available 24 hours a day as the teaching staff can quickly address comments at any time using mobile devices.

\section{Facebook - Negative}

- Administration time is required to add students to the groups.

- The automated email notifications alerting to trivial or irrelevant comments sent by Facebook can be frustrating.

- Some students did not understand the concept of a Facebook group and did not join through fear that all of their personal profile information would be revealed to the group members.

- There is no control over undesirable personal profile pictures or misleading information. For example, a profile picture may feature the student doing an obscene gesture or engaging in something that may offend certain groups of people.

\section{Student Observations}

Quantitative and qualitative evidence were gathered from students regarding their perceptions of the course. The university conducts formal online surveys each semester in the form of Student Evaluations of the Course (SEC) and Student Evaluations of the Teaching (SET). The SEC is regarding the quality of the subject itself and the SET is specific to teaching staff members. The SEC and SET for this course averaged 3.7 and 4.2 out of a possible 5 respectively across all questions. The response rate for the SEC and SET was 38\% (out of 122 students enrolled).

As the response rate for SEC and SET was less than half of the total student cohort and the question structure is rigid (i.e., does not address the attempts at using new technologies towards online group work), an alternative survey was enforced through Blog 4. The students were asked questions specific to the use of the wiki, blogs, Facebook and their general recommendations for how to improve the course (see Table 2). This increased the response rate to $95 \%$ (out of 122 students enrolled). However, there is the possibility that some positive bias may have been introduced, as the alternate survey was part of a student's formal assessment (i.e., students may have wanted to impress the lecturer through providing favourable feedback). An interesting statistic to emerge from the survey was that $51 \%$ of the respondents had already completed an online subject during their studies. This evidences the rise in popularity of online courses and the need for teachers to embrace new technologies and methodologies for online learning.

Table 2: Survey questions gauging the online teaching experience for the preliminary POGIL trial

\#

Text of question

1 Is this your first online subject?

2 What did you enjoy about the use of blogs during the course? (Please provide a few sentences or bullet points.)

3 Did you feel that group work via blogs is a productive way to work through the course concepts (e.g., Web 2.0, information architecture, and content management)? Please indicate on a scale of one to five (where $1=$ Strongly disagree to $5=$ Strongly agree)

$4 \quad$ What did you enjoy about the use of Facebook during the course? (Please provide a few 
sentences or bullet points.)

5 Do you feel that the use of social networking enhanced your sense of being part of the class community given there was no real-time interaction within the subject (i.e., no lectures or tutorials)? Please indicate on a scale of one to five (where $1=$ Strongly disagree to $5=$ Strongly agree)

6 What would you recommend to enhance the experience of a student in an online environment? (Please provide a few sentences or bullet points.)

Figure 2 presents the quantitative statistics regarding student's perceptions of how conducive the use of blogging and Facebook were in enhancing the online learning experience. Figure 2 suggests that the majority of the class did find blogging an interesting way to undertake assessment, or were impartial rather than negative. A teaching staff observation was that the popularity of blogging increased towards the end of the subject as students became more comfortable with the concept of blogging and commenting on other students' blogs. Figure 2 also indicates that the majority of students appreciated the use of Facebook in the course. However, not all of the students joined the group. As such these students either responded as "not applicable" or rated the question low.
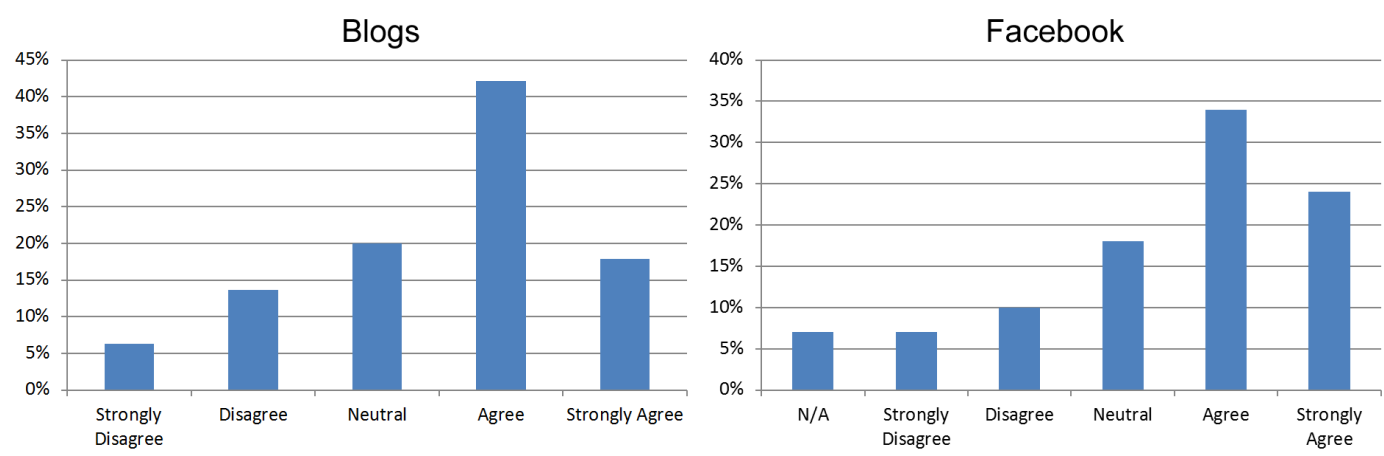

Figure 2. Student perceptions on how conducive the use of blogs and Facebook were to the learning experience throughout the course as per the POGIL survey.

\section{Blogs - Positive}

- $\quad$ Students enjoyed learning something new and different.

- Students enjoyed the flexibility in deadlines.

- Blog work facilitates peer validation while the assessment remains individual.

- Blog work allows for individuality and creativity in the course (less formality).

- Blog work promotes interaction with other students.

- Maintaining a blog allows a student to practice writing skills.

\section{Blogs - Negative}

- The requirement that students had to comment ten other blogs was too large. Smaller predefined groups would be better (as in the philosophy for POGIL).

- There was some resistance to blogs at first. This was largely due to not understanding the purpose of using a blog and general unfamiliarity with blogging.

- There was some resistance to commenting on others' blogs due to principle, not wanting to upset others, or some confusion as what to comment about.

- Some students expressed frustration with other students not keeping up-to-date which limited the ability for interaction via commenting on each other's blog postings.

\section{Class Wiki - Positive}

- Students enjoyed the use of emoticons as a form of feedback to track progress (or lack of progress). 


\section{Class Wiki - Negative}

- The wiki was a navigation bottleneck in that students had to always traverse back to the LMS after making a comment on a blog as the back button in the browser would no longer work.

\section{Facebook - Positive}

- Students appreciated the "physical aspect" in that profile pictures and other personal information put faces to names and made the subject feel a bit more personal - working as a collective group.

- Facebook serves as a central source of information.

- Students found it easy to share ideas, ask questions, comment on others' posts and get almost instantaneous feedback/answers.

- Students appreciated constant updates or clarifications about the course content.

- "Liking" other students' posts if they shared the same view or appreciated a response.

- Students enjoyed seeing what problems other students were having.

- Students found Facebook to be a more relaxed environment than an official class forum.

- The Facebook interface is simple and familiar.

- Facebook has higher reliability and quality of use than a forum hosted on a university server.

\section{Facebook - Negative}

- There were students with moral or ethical objection to using Facebook primarily due to Facebook's dubious terms-of-service, targeted marketing and/or lax privacy policy.

\section{Conclusions}

This paper described a preliminary study for determining a suitable technological platform for delivering POGIL online. A combination of a LMS, wiki, blogs and Facebook were trialled within the curriculum of an online third year IT course. POGIL was condensed to suit the online environment without detracting from the main structure; the peer accountability, student engagement and process-oriented activities were maintained. The goal was to see whether the online technologies were conducive to building a student-centric community and promoting engagement, which are both outcomes of POGIL group work. From the teacher's perspective, this preliminary study found the technologies are potentially suitable to both the challenges of online group work initiatives and achieving learning outcomes. Official institution survey results on the quality of the course and teaching proved favourable. A specific survey targeting student's perceptions of using blogging and Facebook for engagement and group work suggested that students were largely receptive to their use in an online teaching environment.

Future work involves trialling online POGIL using "highly structured" groups by strictly defining the roles of Manager, Recorder, Reflector and Presenter (as in the study by Myers, et al. (2012)). This may require several group members taking on the same role to ensure accountability and redundancy if a group member cannot complete their role. Furthermore, some elements of intergroup policing or peer-based assessment could be used to spark interaction and feedback. Finally, additional future work would be to determine what other ICTs could be used with POGIL. There is the option of using web-based conferencing (e.g., Skype) and online web conferencing tools (e.g., Wimba, Collaborate, etc.) to enhance the student interaction (Challenge 1) but introduces a further challenge in arranging real-time meetings. There is also slightly more technical knowledge required, more powerful computing infrastructure is necessary (Challenge 3), and more reliance placed on technology (Challenge 4). 


\section{References}

Bauersfeld, H. (1995). The structuring of the structures: Development and functioning of mathematics as a social practice. In L. P. Steffe \& J. Gale (Eds.), Constructivism in education (pp. 137-144). Hillsdale, NJ: Lawrence Erlbaum.

Ben-Ari, M. (1998). Constructivism in computer science education. SIGCSE Bulletin, 30(1), $257-$ 261.

Davidson, R. (2012). Wiki use that increases communication and collaboration motivation. Journal of Learning Design, 5(2), 38-49.

Douglas, E. P., \& Chiu, C. (2012, October 8). Process-oriented guided inquiry learning in Engineering. Procedia - Social and Behavioral Sciences, 56, 253-257.

Farrell, J. J., Moog, R. S., \& Spencer, J. N. (1999). A guided-inquiry general Chemistry course. Journal of Chemical Education, 76(4), 570-574.

Gray, H. (2013). Facebook for student success: A preliminary study. Paper presented at the 11th International Conference on Computer Applications, Yangon, Myanmar.

Hanson, D., \& Wolfskill, T. (2000). Process workshops - A new model for instruction. Journal of Chemical Education, 77(1), 120-130.

Hanson, D. M. (2006). Instructor's guide to process-oriented guided-inquiry learning. Lisle, IL: Pacific Crest.

Kearsley, G., \& Shneiderman, B. (1998). Engagement theory: A framework for technology-based teaching and learning. Educational Technology, 38(5), 20-23.

Moog, R. S., \& Spencer, J. N. (2008). Process oriented guided inquiry learning (POGIL). American Chemical Society. New York: Oxford University Press.

Myers, T., Monypenny, R., \& Trevathan, J. (2012). Overcoming the glassy-eyed nod: An application of process-oriented guided inquiry learning techniques in Information Technology. Journal of Learning Design, 5(1), 12-22.

POGIL Process-oriented guided inquiry learning. (2012). Retrieved from http://pogil.org

\section{Acknowledgments}

The authors would like to acknowledge Janelle Prendergast, Peter Bernus and Heather Gray.

Copyright $@ 2013$ Jarrod Trevathan and Trina Myers 